\title{
Relational learning in pigeons: The role of perceptual processes in between-key recognition of complex stimuli
}

\author{
EUAN M. MACPHAIL, MARK GOOD, R. C. HONEY, and ALAN WILLIS \\ University of York, York, England
}

\begin{abstract}
In Experiment 1, we used six procedures in a series of unsuccessful attempts to obtain relational learning using trial-unique pictorial stimuli in pigeons. The Experiment began by testing conventional (three-key) matching-to-sample (MTS) and nonmatching-to-sample (NMTS); in subsequent stages of the experiment we progressively incorporated features of techniques that do obtain relational learning in a single-key apparatus. In Experiment 2, we found that acquisition of NMTS using pictorial stimuli proceeded no more rapidly than acquisition of a conditional discrimination. Experiment 3 showed that acquisition of NMTS was more rapid than acquisition of MTS when plain colored stimuli were used, but not when pictorial stimuli were used. These three experiments suggest that pigeons do not recognize pictorial stimuli shown on different keys. In Experiment 4, between-key recognition was obtained with familiar but not with novel pictorial stimuli. It is argued that perceptual learning facilitates the detection of the between-key identity of complex stimuli, and that perceptual processes may underlie the difficulty in demonstrating relational learning in pigeons.
\end{abstract}

Pigeons find it very difficult to respond appropriately according to whether two simultaneously presented stimuli are the same or different. In matching-to-sample (MTS) or nonmatching-to-sample (NMTS) tasks, pigeons are able to learn to peck the side-key stimulus that is the same as (or different from) the sample stimulus exposed on the center key. However, only two alternative stimuli are used in conventional (N)MTS tasks, and when pigeons are subsequently tested with two novel stimuli, little or no evidence of positive transfer is obtained (e.g., Wilson, Mackintosh, \& Boakes, 1985a). Such findings suggest that pigeons, rather than achieving relational learning (choosing, that is, according to the relationship, same or different from sample, that holds for the choice stimuli), solve these tasks as conditional "if-then" discriminations (selecting one stimulus when a given sample is shown and the other when the alternative sample is shown). When trial-unique stimuli (stimuli that are shown at the most only once per session) are used, a solution in terms of (multiple) conditional discriminations becomes very difficult, and pigeons have been shown to achieve relational learning, showing significant

This research was supported by a grant from the U.K. Medical Research Council. M.G. is now at the Department of Pharmacology, University of Edinburgh Medical School, 1 George Square, Edinburgh EH8 9JZ. R.C.H. is now at the Sub-Department of Animal Behaviour, Madingley, Cambridge CB3 8AA. Correspondence concerning this article should be addressed to E. M. Macphail, Department of Psychology, University of York, York, YO1 5DD, England (e-mail: emm1@ york.ac.uk).

-Accepted by previous editor, Vincent M. LoLordo positive transfer to entirely novel stimuli in a samenessdifference discrimination as well as an MTS task (Santiago \& Wright, 1984; Wright, Cook, Rivera, Sands, \& Delius, 1988). But the acquisition of these tasks using trial-unique stimuli required many thousands of training trials and encouraged the conclusion (e.g., Mackintosh, 1987) that pigeons are not readily capable of abstracting the generalized sameness-difference concept.

The results of experiments involving a choice between simultaneously presented stimuli stand in sharp contrast to those reported by Macphail and Reilly (1989), in which novel pictorial stimuli were shown on a single key. Each stimulus was shown on only two occasions throughout the experiment, and the pigeons rapidly learned to respond at a higher rate to a stimulus on its first presentation (which was followed by reward) than on its second (nonrewarded) presentation. In Experiment 4 of Macphail and Reilly's report, for example, there were 48 trials per session, and each of 24 entirely novel slides was shown twice in each session. From Session 4 onward (i.e., following completion of fewer than 150 trials), all 5 pigeons showed a higher mean rate of response to novel slides than to second presentations of those same slides. Mackintosh (1987) has argued that the essential requirement of the generalized MTS task is precisely the discrimination between novelty and familiarity - the stimulus that matches the sample is familiar; the nonmatching stimulus is novel. Accordingly, an animal that learns rapidly to base its responding on novelty versus familiarity should be expected to master generalized (N)MTS tasks equally rapidly. In the experiments described in the present re- 
port, we attempted to resolve this paradox by using apparatus and materials that were comparable to those used by Macphail and Reilly.

\section{EXPERIMENT 1}

One obvious possibility is that some aspect of the apparatus used by Macphail and Reilly (1989) was peculiarly well suited to the detection of sameness and difference; the pigeons in that study pecked directly at keys onto which colored slides were back-projected. Santiago and Wright (1984) used colored slides, but the screens onto which they were projected were some distance $(61.5 \mathrm{~cm})$ from the pigeons; although the pigeons in Wright et al. (1988) pecked directly at pictures on a screen, the pictures were cartoons of line drawings that had considerably less detail than conventional photographic slides of objects and natural scenes. In Experiment 1 , therefore, we began by exploring the question of whether pigeons can rapidly acquire generalized (N)MTS, using techniques similar to those of Macphail and Reilly. The various phases of the experiment consisted of a series of (universally unsuccessful) procedural variations that were introduced in response to failures to obtain relational learning.

In Phase 1 we used a conventional design in which the sample stimulus was exposed on the center key for $5 \mathrm{sec}$. Following this, all three keys were illuminated until a single response was made to one of the two side keys. Trials on which an incorrect choice was made were repeated until the correct choice occurred. We thought that failure to obtain successful discrimination in Phase 1 might have been due to the use of a correction procedure, which means that, on trials on which an error has been made, the correct stimulus will not be entirely novel. In Phase 2 we assessed the potential importance of this factor by omitting correction trials, but we also failed to obtain successful discrimination. Since reliable discrimination was obtained by Macphail and Reilly (1989) following 150 trials, and because we were interested only in knowing whether rapid acquisition could be demonstrated, we terminated Phases 1 and 2 after 450 trials.

In Phases 1 and 2, the sample stimulus remained illuminated during the choice phase, and it seemed possible that this aspect of the procedure detracted from the salience of the contrast in familiarity between the matching and nonmatching stimuli; perhaps, for example, the matching stimulus would not be categorized as familiar (seen previously) if the sample was still present. In Phase 3, therefore, we explored acquisition of zerodelay (N)MTS. Phase 3 was terminated after 300 trials, thus allowing more than twice as many trials as had been sufficient to obtain reliable discrimination in Macphail and Reilly (1989).

Following the failure of Phases 1-3 to reveal successful discrimination, in Phases 4-6 we introduced procedures in which no choice was involved and a successive go/no-go measure of discrimination was used, as in the original Macphail and Reilly (1989) study. Over these final three phases, each of which was terminated after a minimum of 300 trials, we modified our procedures step by step so that they would resemble more closely those used by Macphail and Reilly in the expectation that eventually successful discrimination must be achieved. In Phase 4, following offset of the sample stimulus on the center key, the right key was illuminated until a single response had occurred (or until a fixed time had elapsed). We assessed discrimination by comparing latencies on trials on which the sample had been reexposed on the side key with those on trials on which a novel stimulus was shown. In Phase 5 we moved another step closer to Macphail and Reilly's procedure by using, in place of the simple latency measure of Phase 4, the graded response rate measure used in the previous study.

In the first five phases of the experiment, responding to the sample stimulus was without effect, and the sample was shown for $5 \mathrm{sec}$ before the test stimuli (or stimulus) were illuminated. The first showing of a slide in Macphail and Reilly's (1989) procedure is analogous to the showing of a sample stimulus in the present experiments, and in that study responding to novel slides was rewarded according to a fixed-interval 10 -sec (FI 10-sec) schedule, so that the first response after $10 \mathrm{sec}$ obtained food. In a further attempt to converge on the procedures used in the novelty/familiarity discrimination, the sample stimulus was exposed in Phase 6 for a minimum of $10 \mathrm{sec}$, and responding to it was rewarded according to an FI 10-sec schedule. It seems at least plausible that detection of familiarity might be enhanced by a longer sample duration, or that attention to the sample might be enhanced by the availability of reward.

\section{Method}

\section{Subjects}

Thirteen pigeons (Columba livia) were used; they had had previous experience that involved acquisition of autoshaping in a different apparatus. Before training began, they were gradually reduced to $80 \%$ of their ad-lib-feeding weights (range, 395-560 g) by food deprivation and were maintained at that level throughout the experiment. The birds were housed individually in cages in which water was always available.

\section{Apparatus}

The subjects were trained in a pigeon chamber $(35 \times 35 \times$ $35 \mathrm{~cm}$ ) that contained three square $4 \times 4 \mathrm{~cm}$ response keys. The center key was mounted directly over a grain feeder at a height of $25 \mathrm{~cm}$ above the floor. The remaining two keys were mounted on either side of the center key, at the same level. The keys were $5 \mathrm{~cm}$ apart, center to center, and behind each key was a flap that, when operated, prevented transillumination of the key. All three keys could be transilluminated by a Rollei projector located $55 \mathrm{~cm}$ behind the front wall of the apparatus, and each $6 \times 6 \mathrm{~cm}$ slide in the projector tray contained three images, arranged so that a different $3.9 \times 2.7 \mathrm{~cm}$ image was projected onto each of the three keys. There was a houselight in the ceiling of the chamber. The chamber was located in a quiet, darkened room, and background white noise was provided by a fan in the chamber.

The projector and the sequence of events in the chamber wero controlled on line by a Nova 3 computer that also collected re- 
sponse data. Programs were written in the Act- $\mathrm{N}$ language (Millenson, 1971).

\section{Materials}

The 450 slides used in this Experiment were prepared from a wide variety of different photographic $5 \times 5 \mathrm{~cm}$ color transparency slides whose subjects were principally exterior scenes, but that also included interior scenes, objects, faces, and so on. Each slide was projected onto a specific region of unexposed film, and the resulting $6 \times 6 \mathrm{~cm}$ slide contained three images, located so that when subsequently projected, the three original scenes would be shown on the three keys. The long axes of the images could be horizontal or vertical, and slides were shown in their normal orientation. The images chosen for display on any given day were scanned to ensure that no two images were obviously similar, but no systematic effort was made to check for similarity of images to any shown on previous days.

\section{Procedure}

The birds were first trained to eat from the feeder and then, by the use of an autoshaping procedure (Brown \& Jenkins, 1968), to peck the center, left, and right keys when a yellow rectangle was projected onto any one of them. Seven birds were assigned to an NMTS group, and 6 were assigned to an MTS group.

Phase 1: Conventional (N)MTS, correction procedure. Each trial began with the illumination of the center key with the sample image - an image that had not previously been seen. After $5 \mathrm{sec}$, all three keys were illuminated; one side key showed an image that matched the image on the center key, and the other side key showed a novel image that did not match the center-key image (and would not be seen again). A single response to the side key showing the matching (nonmatching) image obtained 4-sec access to food in the hopper; a single response to the nonmatching (matching) key resulted in a 4-sec time-out (TO). Following this, the trial was repeated, showing the same sample and choice images in the same locations. Intertrial interval (ITI) duration was $4 \mathrm{sec}$, and the houselight was illuminated throughout, except during hopper operation or TOs. Center-key responses during either the sample or the choice phase had no effect. Trials were organized according to Gellerman sequences so that the matching stimulus did not appear more than three times in succession (ignoring correction trials) on the same side key. There were 30 trials (excluding correction trials) in each session, and there were 15 training sessions.

Phase 2: Conventional (N)MTS, noncorrection procedure. The procedure was identical to that of Phase 1, except that correction trials did not occur following incorrect choices; slides previously used in Phase 1 were recycled as novel slides in both this and succeeding phases of the experiment. Throughout Phases 2-6 of the experiment, then, slides described as novel had in fact been seen before, but were trial unique and had not been seen for a minimum of 15 sessions. One bird from the MTS group abandoned responding after seven sessions and could not be induced to resume. Its data were therefore discarded, and the MTS group was reduced to $N=5$. Phase 2 ran for 15 sessions.

Phase 3: Zero-delay (N)MTS. The procedure was identical to that of Phase 2, except that the center key was extinguished when the side keys were illuminated. Phase 3 ran for 10 sessions.

Phase 4: Successive (N)MTS, latency measure. The procedure was identical to that of Phase 3, except that the offset of the sample image on the center key was followed by the presentation of a test image shown on the right side key only. The side key remained illuminated during positive as well as negative trials until a single response was made to it, or for a maximum of either $5 \mathrm{sec}$ (Sessions 1-4) or $10 \mathrm{sec}$ (Sessions 5-14). Responses to the left key were not recorded and had no consequences. For the MTS group, positive trials were trials on which the side key showed a slide that matched the preceding sample slide, and negative trials were trials on which a nonmatching slide was shown (vice-versa for the
NMTS group). Positive trials ended in reward, whether a response had occurred or not, and the maximum duration of trials was increased after the fourth session because 2 birds in fact made relatively few responses throughout Sessions $1-4$. All the subjects responded on virtually all trials from Session 5 onward. There were 14 sessions in all.

Phase 5: Suceessive (N)MTS, rate measure. The procedure was identical to that of Phase 4 , except that side-key illumination persisted for a minimum of 10 sec. Positive trials were arranged according to an FI 10-sec schedule, so that the first response to the side key after $10 \mathrm{sec}$ obtained food; negative trials terminated after a minimum of $10 \mathrm{sec}$, but subject to the condition that $3 \mathrm{sec}$ had elapsed without a response. Phase 5 ran for 10 sessions.

Phase 6: Successive (N)MTS, sample rewarded. The subjects, apparatus, and procedure were identical to those of Phase 5, except that responding to the center-key sample was rewarded according to an FI 10-sec schedule; where food had not been obtained within $20 \mathrm{sec}$ of sample onset, the center key was extinguished and "free" food was delivered. Immediately following 4-sec access to the hopper, the right side key was illuminated with the test image. Phase 6 ran for 10 sessions.

\section{Results}

\section{Phases 1-3}

Table 1 shows the mean number of correct choices of the two groups on the final session of each of the first three phases. None of the scores were significantly greater than chance (15 correct choices) level (twotailed $t$ test, all $p s>.05$ ). Although not significantly above chance level, the performance of Group NMTS on Day 15 of Phase 1 did approach significance (mean of 17.7 correct, $.06>p>.05$; all other $p s>.1$ ) and suggested the possibility that discrimination might be emerging. However, the mean correct scores of Group NMTS for the final five sessions were $15.1,17.1,13.0$, 15.4 , and 17.7 , so there was in fact no evidence of any steady improvement. The mean correct-choice score for this group over the final five sessions was 15.7 , and this also was not significantly better than chance $[t(6)=$ $1.37, p>.2]$.

\section{Phases 4-6}

Performance in Phase 4 was assessed by measuring the latency of response to the side key, taken to be $10 \mathrm{sec}(5 \mathrm{sec}$, over Sessions 1-4) on those trials on which no response occurred. Performance in Phases 5 and 6 was assessed by obtaining discrimination ratios (DRs: the ratio of the number of responses in the first $10 \mathrm{sec}$ of positive trials to the total number of responses in the first $10 \mathrm{sec}$ of both positive and negative trials). The performance of the two groups on the final sessions of Phases 4-6 is summarized in Table 2. The

Table 1

Mean Number of Correct Choices (Out of 30) on the Final Session of Phases 1-3 in Experiment 1

\begin{tabular}{lccc}
\hline & Phase 1 & Phase 2 & Phase 3 \\
Group & Day 15 & Day 15 & Day 10 \\
\hline MTS & 13.8 & 15.4 & 14.2 \\
NMTS & 17.7 & 16.0 & 15.6 \\
\hline
\end{tabular}

Note--MTS, matching-to-sample; NMTS, nonmatching-to-sample. 
Table 2

Mean Latencies (Phase 4; in Seconds) and Discrimination Ratios (DR, Phases 5 and 6 ) for the Final Sessions of Phases 4, 5 , and 6 in Experiment 1

\begin{tabular}{|c|c|c|c|c|}
\hline \multirow[b]{3}{*}{ Group } & \multicolumn{2}{|c|}{ Phase 4, Session 14} & \multirow{2}{*}{$\begin{array}{l}\text { Phase 5, } \\
\text { Session } 10\end{array}$} & \multirow{2}{*}{$\begin{array}{l}\text { Phase 6, } \\
\text { Session } 10\end{array}$} \\
\hline & Positive-Trial & Negative-Trial & & \\
\hline & Latency & Latency & $\mathrm{DR}$ & DR \\
\hline MTS & 0.79 & 0.84 & .50 & .49 \\
\hline NMTS & 1.27 & 1.10 & .51 & .50 \\
\hline
\end{tabular}

Note-MTS, matching-to-sample; NMTS, nonmatching-to-sample.

difference between the positive- and negative-trial latencies on Session 14 of Phase 4 was not significant for either group (two-tailed $t$ tests, both $p \mathrm{~s}>$.2], and none of the four mean DRs obtained on the final days of Phases 5 and 6 differed significantly from chance $(.50)$.

\section{Discussion}

The successive failures of the phases of this experiment increasingly tended to surprise us. There seemed to be very few differences between the procedures of Phase 6, for example, and those of Macphail and Reilly (1989). In each case, novel slides were rewarded according to an FI 10 -sec schedule, and familiar slides (slides previously seen briefly) were not rewarded. The design of Phase 6 had the consequence that responding to $75 \%$ of the slides shown was rewarded (all of the samples, and 50\% of the slides shown on the side key), but it does not seem likely that the rich reward schedule should have a drastic effect on discrimination. Macphail and Reilly used somewhat longer retention intervals (minimum $8 \mathrm{sec}$ ), but it seems even less likely that the short retention intervals of Phase 6 (in which the onset of test stimuli was separated from the offset of the sample stimuli only by the 4-sec duration of the feeder operation) should impair acquisition.

One obvious major difference between the procedures used in Experiment 1 and those employed by Macphail and Reilly (1989) was that both side and center keys were in use in Experiment 1, whereas Macphail and Reilly used only the center key. To perform successfully in any of the phases of Experiment 1 , the pigeons had to detect the identity of images shown on different keys; in Macphail and Reilly's (1989) study, the requirement was to detect the identity of images shown on the same key. One possible interpretation of the entire pattern of results that we obtained, then, is that although pigeons find it easy to detect the identity of pictorial stimuli shown twice on the same key, they find it very difficult to detect the identity of pictorial slides shown on two separate keys. A difficulty facing confident acceptance of this conclusion is, however, that there are phenomena that indicate that pigeons may readily detect between-key identity of stimuli. We explored two such phenomena in Experiments 2 and 3.

\section{EXPERIMENT 2}

There have been reports (e.g., Wilson et al., 1985a) that acquisition of NMTS is more rapid than acquisition of conditional discriminations in which there is a wholly arbitrary relationship between the stimulus shown on the center key and the reward value of the two stimuli between which a choice is made on the side keys. Such findings imply that pigeons do detect the relationship between two identical images shown on different keys. One potentially important feature of the Wilson et al.'s (1985a) procedure is that they used colors as stimuli. It is possible that pigeons' perception of plain colored stimuli will be relatively uninfluenced by the key on which they are displayed, and in Experiment 2 we asked whether a superiority of NMTS over conditional discrimination will emerge when pictorial images are used.

\section{Method}

\section{Subjects}

Sixteen experimentally naive pigeons (Columba livia) were used. The Experiment was run in two identical replications. The ad-lib-feeding weights of the 8 birds used in Replication 1 ranged from 350 to $480 \mathrm{~g}$, and those of the 8 birds used in Replication 2 ranged from 380 to $445 \mathrm{~g}$. Deprivation procedures and housing conditions were the same as those of Experiment 1.

\section{Apparatus}

The apparatus was that used in Experiment 1, and the slides were prepared in the same way. They contained combinations of images drawn from a total of only four scenes, referred to here as "garden," "birds," "arch," and "beach." The slides used in the NMTS task each showed a center-key image that matched either the left- or the right-key image; the slides used in the conditional task each showed three different images.

Pretraining. The birds were trained to eat from the feeder and to peck the center and side keys, using the procedures of Experiment 1 .

Training. Each trial began with the illumination of the center key for $5 \mathrm{sec}$, during which time responses were without effect. After $5 \mathrm{sec}$, all three keys were illuminated, and a single response to either side key constituted a choice. If the choice was correct, the food hopper was operated for $4 \mathrm{sec}$; if the choice was incorrect, there was a 4-sec TO. Correct trials were followed by a 4-sec ITI; incorrect trials were followed by the 4-sec ITI and a correction trial that was identical to the initial noncorrection trial.

Two of the birds in the NMTS task saw slides that contained garden and birds images, and the correct key was that showing an image that did not match the center-key image; the other 2 birds in this task saw arch and beach images. Two of the birds in the conditional task saw either a birds or a garden image on the center key, and they had to choose between an arch and a beach image on the side keys; the correct side key was arch when birds was shown on the center key, and beach when garden was shown. The other 2 birds in the conditional task saw either a beach or an arch image on the center key, and they had to choose between a garden and a birds image on the side keys; the correct side key was garden when beach was shown on the center key, and birds when arch was shown.

The two center-key images alternated every (noncorrection) trial. The correct side key for a given trial was organized according to Gellerman sequences, so that the same key was not correct for more than 3 (noncorrection) successive trials, and each key 


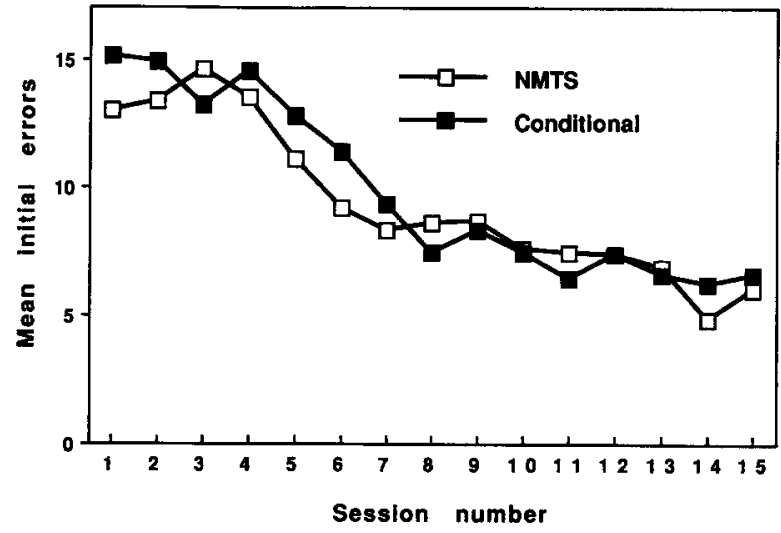

Figure 1. Experiment 2: Mean initial errors (maximum 30) for the 15 sessions of the nonmatching-to-sample (NMTS) and conditional tasks.

was correct on 5 of every 10 trials. Each of the 15 sessions contained 30 noncorrection trials, and the houselight was illuminated only during the ITI.

\section{Results}

Inspection of the data showed that performance was similar in both replications; Figure 1 summarizes the results of the experiment, pooled across replications. The figure shows that the birds in both tasks showed a steady improvement, and that performance was similar in the two tasks. Analysis of variance (ANOVA) of the data summarized in Figure 1 showed a significant effect of session $[F(14,196)=28.06, p<.001]$; neither the effect of group nor the interaction was significant (both $F \mathrm{~s}<1$ ).

Although the group $\times$ session interaction did not approach significance, inspection of Figure 1 shows that the birds that performed the NMTS task showed somewhat fewer errors than those that performed the conditional task over the early sessions. To provide a further check that those early differences were not reliable, we carried out (1) an ANOVA confined to the data from the first six sessions only, and (2) a $t$ test on the Session 1 scores of the two groups. Neither analysis showed a significant difference between the groups (both $p \mathrm{~s}>.1$ ).

\section{Discussion}

The absence of any superiority of the NMTS over the conditional task is surprising given the results obtained by Wilson et al. (1985a), but is congruent with the proposal that NMTS was treated by the pigeons as a conditional discrimination. The results of Experiment 2 are, then, those that would be anticipated if the pigeons did not detect the relationship between a pictorial image seen on the center key and the same image seen on a side key. We did not, however, run a condition in which colored stimuli were used, and it remains possible that some feature of our procedure militates against relational learning, independently of the stimuli used. In
Experiment 3 we explored a related phenomenon and directly contrasted performance using colored stimuli with performance using pictorial stimuli.

\section{EXPERIMENT 3}

There have been reports (e.g., Ginsburg, 1957; Wilson et al., 1985a) that pigeons learn NMTS tasks more rapidly than MTS tasks when plain colored stimuli are used. If patches of light on the center key were always regarded as different from patches of light on side keys, there would be no basis on which a difference between an NMTS and an MTS task could be explained. These reports, therefore, like reports of superiority of NMTS over conditional task acquisition, imply that pigeons do detect the identity of patches of color shown on different keys. In Experiment 3 we asked whether a superiority of NMTS over MTS would be found by using color stimuli in our apparatus, and whether a similar effect would emerge when pictorial stimuli were used. A within-subjects design was used to improve the sensitivity of the choice data. Two versions of the experiment were carried out. In the first version, the birds learned two NMTS tasks-one using colored stimuli and the other using pictorial stimuli. These birds then went on to learn two MTS tasks, using different colored and pictorial stimuli. In the second version, the birds first learned two MTS tasks, and then went on to learn two NMTS tasks.

\section{Method}

\section{Subjects and Apparatus}

The 8 birds used in the first version had previously served as the subjects in the first replication of Experiment 2 . The 8 birds used in the second version were experimentally naive; their ad-lib-feeding weights ranged from 300 to $560 \mathrm{~g}$. Deprivation procedures, housing conditions, and apparatus were the same as those for Experiments 1 and 2 .

\section{Procedure: Version 1}

Phase 1: Acquisition of NMTS. Trials were organized in the same way as those in the NMTS task of Experiment 2. In one condition (COL), the slides used showed a plain color on each key (either red on the center key and a red and a green side key, or green on the center key and a red and a green side key); in the other condition (PIC), two pictorial images (Harbour and York University), neither of which had previously been seen by the birds, were used in place of red and green.

Each bird served as a subject in both conditions. Half of the birds performed the COL condition in the morning, and the PIC condition in the afternoon; the remaining birds performed the conditions in the reverse order. This phase of the experiment ran for two 30-trial sessions.

Phase 2: Acquisition of MTS. The procedures used in Phase 2 were generally identical to those used in the NMTS task, except that (1) reward was available only for responses to the matching key, and (2) for the COL condition, the stimuli used were blue and yellow, and for the PIC condition, two novel images were used (house and snow scene).

The groups continued to perform the same types of discrimination (PIC or COL) in the morning and the afternoon as in Phase 1, and there were two sessions in this phase of the experiment. 
Table 3

Mean Initial Errors (Maximum, 60) Pooled Over the Two Sessions of Acquisition of the NMTS and MTS Tasks in Experiment 3

\begin{tabular}{ccccc} 
& \multicolumn{3}{c}{ NMTS } & \multicolumn{2}{c}{ MTS } \\
\cline { 2 - 5 } Version & COL & PIC & COL & PIC \\
\cline { 2 - 6 } 1 & 22.9 & 29.3 & 28.8 & 29.6 \\
2 & 23.0 & 29.3 & 27.3 & 31.1 \\
\hline
\end{tabular}

Note-NMTS, nonmatching-to-sample; MTS, matching-to-sample; COL, color condition; PIC, picture condition.

\section{Procedure: Version 2}

Pretraining. The birds were trained to peck all three keys using the same techniques as those used in Experiment 1.

Phase 1: Acquisition of MTS. The procedures used were identical to those used in Phase 2 of Version 1, except that the stimuli used for the COL condition were red and green.

Phase 2: Acquisition of NMTS. The procedures used were identical to those used in Phase 1 of Version 1, except that the stimuli used for the COL condition were blue and yellow.

\section{Results and Discussion}

Inspection of the initial error scores showed no systematic effect of the order (a.m. vs. p.m.) in which the conditions were performed, and the data for each version have, accordingly, been pooled across all the birds. The results of the experiment are summarized in Table 3 . In the COL condition, acquisition of NMTS showed considerably fewer errors than acquisition of MTS, but there was little difference among the scores for the PIC condition-none of which showed much deviation from chance (30 errors) level. An ANOVA of the data for the COL condition (with version and task, MTS vs. NMTS, as factors) showed a significant effect of task $[F(1,14)=$ $6.95, p<.02$ ]; neither version nor the interaction achieved significance both $(F \mathbf{s}<1)$. An ANOVA of the PIC condition data showed no significant effects (all $p s>.3$ ).

No significant effects associated with version were found, and Table 3 shows, in fact, very similar scores for the two versions. The implication is that neither the differing previous experience of the birds nor the order in which MTS and NMTS were experienced had any significant effect. The fact that no negative transfer occurred following a shift from MTS with one set of stimuli to NMTS with a novel set replicates a similar finding by Wilson et al. (1985a). Those authors did, however, find negative transfer following a shift from NMTS to MTS training; the fact that no such effect was seen in the present experiment may reflect the fact that performance in the NMTS task had not in fact reached a high level of accuracy with either pictorial or color stimuli after only two training sessions.

The faster acquisition of the NMTS (relative to the MTS) task when colored stimuli were used confirms previous work (e.g., Ginsburg, 1957; Wilson et al., 1985a). When the data for Session 1 of the color NMTS task (of both versions) were examined, there was evidence that the superiority of NMTS over MTS reflected an initial preference for the nonmatching stimulus. An ANOVA of those data showed a significant main effect of task $[F(1,14)=84.50, p<.03]$; neither the main effect of version nor the version $\times$ task interaction approached significance (both $p \mathrm{~s}>.2$ ). The mean number of errors in the NMTS task was 13.3 , and this was significantly better than chance $[15 ; t(15)=2.41, p<.03]$. But what is more pertinent here is that the corresponding score for the MTS task was 16.6, and that, despite the fact that over the 30 trials of Session 1 the nonmatching stimulus was not rewarded, this score was worse than chance at a level that approached conventional significance $[t(15)=1.90, .05>p<.08]$. The clear implication of this pattern of performance is that pigeons choosing from a display of novel colors show an initial preference for a nonmatching color, even if that stimulus is not rewarded; a similar initial preference for the nonmatching stimulus in a color MTS task has been reported by Zentall and Hogan (1974) and Farthing and Opuda (1974). The source of this preference is not understood (see Wilson et al., 1985a), but it does provide clear evidence that pigeons do readily detect the identity of novel colors shown on different keys.

There was no significant difference between the MTS and NMTS tasks when pictorial stimuli were used. However, in neither case did performance over the two sessions show evidence of learning, and there was a slight trend toward superior performance in the NMTS task. This raises the possibility that further training might have revealed an eventual superiority of NMTS over MTS for pictorial stimuli. There are good reasons for rejecting this possibility. First, Experiment 2 showed that, over an extended training period, performance in an NMTS task did not differ significantly from that in a conditional discrimination; Wilson et al. (1985a), using designs similar to those used here, found in two experiments no significant difference in numbers of sessions to criterion between an MTS and a conditional discrimination. Second, when an advantage for NMTS over MTS training has been reported, that advantage has tended to be most marked over the early sessions of training (Cumming \& Berryman, 1965; Zentall \& Hogan, 1974).

The results of Experiment 3 are, then, congruent with the notion that pigeons do not detect the identity of pictures exposed on two different keys, but do detect the identity of plain colors exposed on different keys.

\section{EXPERIMENT 4}

The results of the preceding three experiments can all readily be accommodated by the proposal that pigeons do not recognize the identity of pictorial slides exposed on different keys. In Experiment 4, we return to the issue raised in Phase 6 of Experiment 1, namely, whether pigeons can master a novelty/familiarity discrimination when the two presentations of slides are on different keys. A within-subjects design was adopted so that performance could be simultaneously assessed 
when the two presentations of slides were on the same key.

Todd and Mackintosh (1990) found that performance using thoroughly familiar slides in a relative recency task was superior to that obtained using entirely novel slides in a novelty/familiarity discrimination. The same set of slides was, accordingly, used for every session in Phase 1 of this experiment. We hoped that the daily reuse of slides would lead to a high level of accuracy on trials on which images were reexposed on the same key, and that the anticipated failure to achieve better-than-chance performance with re-presentations on a different key would be that much more striking.

We used a simple procedure for ordering trials: every block of four trials consisted of two exposures of novel slides followed by the reexposure of the same slides, in the same order. This double-alternation sequence of two rewarded trials followed by two nonreward trials was one that we believed pigeons were unlikely to learn, but that allowed a consistent short retention interval between initial and second presentations of slides; it also has the property that the probability of a rewarded trial is the same following a rewarded and a nonreward trial. After separate training on the double-alternation sequence on the center and the side keys, random sequences were used to determine the key on which slides were exposed, so that initial presentations were equally likely to occur on the center and on the side key, and second presentations were equally likely to occur on the same and on a different key from that used for original presentation.

Test sessions were run to assess the possibility that pigeons might achieve above-chance performance by learning the double-alternation sequence. Because, in fact, the pigeons did achieve successful between-key recognition in the relative recency task of Phase 1 , we used entirely novel slides for each session in Phase 2 of the experiment.

\section{Method}

\section{Subjects}

The subjects were 5 pigeons (Columba livia) that had previously served as subjects in the first replication of Experiment 2 and in Experiment 3; none of the slides used in those experiments were reused in this experiment. Ad-lib weights of the birds ranged from 365 to $475 \mathrm{~g}$, and maintenance and deprivation conditions were the same as those in Experiments 2 and 3.

\section{Apparatus and Materials}

The apparatus was that used in the previous experiments, and the slides were taken from the same collection. None of the images had previously been seen by the birds. The left side key was not used in this experiment; it was not illuminated at any stage, and responses to it were not recorded.

\section{Procedure}

Center-key pretraining. The previous experience of the birds ensured that they pecked reliably to the projection of an image on either key, and center-key pretraining used the following procedure throughout. Each trial began with the illumination of the center key with an image. On positive trials, responding to the center key was rewarded with 4-sec access to the grain feeder according to an FI 10-sec; when a bird failed to gain reward within $20 \mathrm{sec}$ of illumination of the center key, the trial terminated with "free" reward. On negative trials, the center key was illuminated for a minimum of $10 \mathrm{sec}$, no reward was obtained, and the trial ended in a 4-sec TO; negative trials did not terminate until at least $3 \mathrm{sec}$ had passed without a center-key response. The houselight was illuminated during the 2-sec ITI, but not when either key was lit, nor during feeder operation or TO.

A double-alternation sequence was used, so that of each block of 4 trials, the first 2 were positive trials and the second 2 were negative trials. Images shown on the center key on positive trials had not previously been shown on that day; images shown on negative trials were the second showings of images that had first been seen 2 trials previously. The slide tray was loaded with the same 24 slides in a different quasi-random order each day, and tray movements occurred during the hopper operation or TO that ended each trial. There were 1548 -trial sessions with a minimum intersession interval of $24 \mathrm{~h}$.

Side-key pretraining. The procedure for side-key pretraining was identical to that used for center-key pretraining, except that only the right side key was used. Pretraining continued, using the same set of slides (and thus showing the same 24 images) that had been used for center-key pretraining, for 10 sessions.

Training: Phase 1, familiar slides. Trial sequences continued to be arranged according to the double-alternation principle, but images could be shown on either the center or the side keys. For a 4trial block in which each trial could be either a center- or a sidekey trial, there were 16 possible orders of center- and side-key trials. All 16 possible orders were selected randomly (without replacement) so that each order was represented once in a 64-trial sequence; there continued to be 48 trials each day, and three random 64-trial sequences were generated and used consecutively so that each sequence was completed once over the course of four sessions. There were two cycles of four sessions, each of which used the same three 64-trial sequences, in the same order. The 24 slides that had been used in pretraining were used.

Training: Phase 2, novel slides. The procedure used in each session was identical to that used in the training sessions of Phase 1 , except that the slide tray was loaded with entirely novel slides each day. Phase 2 consisted of three four-session cycles; in each cycle, the three 64-trial sequences of Phase 1 were reused to determine the order of side- and center-key illumination.

Test sessions. The procedure for the test sessions was identical to that used for training sessions, except that the tray advanced one position every trial, so that each image was shown only once. Since there were only 24 slides in all, test sessions consisted of 24 trials. The first test session used the center- and side-key ordering used for the first 24 trials of the first session of the four-session training cycle (Trials $1-24$ of the first 64-trial sequence); it took place on the day after the first training session of Phase 1 . The second test session used the trial ordering used for the first 24 trials of the fourth session of the cycle (Trials $17-40$ of the third 64-trial sequence) and was carried out after the final (eighth) training session of Phase 1 . The final test session followed the completion of Phase 2, and 24 more novel slides were shown; the ordering of center- and side-key slide presentations was the same as that used for the second test session.

\section{Results}

\section{Pretraining}

The birds rapidly learned to discriminate between the first and second showings of slides within a session, and the group mean DR for Day 15 of center-key pretraining was .65 ; this score was significantly better than chance $[.50 ; t(4)=2.98, p<.05$, two-tailed]. The corresponding DR for Day 10 of side-key pretraining was .73, and 
this score was also better than chance $[t(4)=6.47, p<$ .005 , two-tailed]. The Day 10 side-key DR was significantly higher than that for the final day of center-key pretraining $[t(4)=4.57, p<.02$, two-tailed]. We assume that the difference between the center- and side-key DRs simply reflected improvement with further training, rather than some intrinsic superiority of side-key presentations, and this assumption is borne out by training performance in which, as will be seen, performance on the two keys was comparable.

\section{Training and Test Sessions}

Phase 1, familiar slides. Performance was assessed by obtaining DRs for three types of positive- and negativetrial pairs - center trial pairs on which a slide was shown on the center key on both its exposures; side pairs on which a slide was shown on the right key on both exposures; and different pairs on which a slide was shown on different keys on its two exposures (either shown first on the center key and second on the right key, or viceversa). DRs were obtained for the two complete foursession cycles, each of which contained three different 64-trial sequences.

The group mean center pair DR for Cycle 1 was .67, and for Cycle 2 it was .71; the corresponding side DRs were .72 and .72 , and the different DRs were .60 and .58 . An ANOVA of the DR data showed a significant effect of pair type $[F(2,8)=26.57, p<.001]$, but neither the main effect of cycle nor the interaction was significant (both $F \mathrm{~s}<1$ ). Subsequent orthogonal contrasts showed that the center- and right-key DRs did not differ significantly $[F(1,8)=3.35, p>.1]$, and that the mean of those two same-key DRs was significantly greater than the different-keys DR $[F(1,8)=49.79, p<.001]$.

Inspection of the results obtained in Session 1 of the first cycle revealed that, contrary to our expectations, the birds had performed at a better-than-chance level on different trial pairs. It was for that reason that we then ran the first test session. The group mean DR on that session was .53 , a score that did not differ significantly from chance $[.50 ; t(4)=1.21, p>.2]$. The mean DR for the second test session was .51, a score that, again, did not differ significantly from chance $(t<1)$.

Although not significantly better than chance, each group test session score was in fact above .50 , and the mean group test score (pooled over the first two test sessions) provides the safest standard against which to test Phase 1 discriminative performance. In three separate two-tailed $t$ tests, the mean center-key DR (.69) was shown to be significantly higher than the mean test DR $[.52 ; t(4)=4.02, p<.02]$, as were the mean side-key DR $[.72 ; t(4)=6.03, p<.005]$ and the mean different-key DR $[.59 ; t(4)=2.95, p<.05]$.

Training: Phase 2 . The birds adapted readily to the use of novel slides and showed no sign of an improvement in performance across the three cycles; the group mean DR (pooled across the three types of trial pairs) was .60 for Cycle 1 and .61 for Cycles 2 and 3. An ANOVA carried out on the DRs for each cycle showed a main effect of pair type $[F(2,8)=10.24, p<.01]$; neither the main effect of cycle nor the interaction was significant (both $p s>.2$ ). The group mean DRs, pooled across the three cycles, were .66 for the center-key pairs, .62 for sidekey pairs, and .53 for different-key pairs. Subsequent orthogonal contrasts showed that the center- and rightkey DRs did not differ significantly $[F(1,8)=1.83, p>$ $.2]$, and that the mean of those two same-key DRs was significantly greater than that of the different-keys DR $[F(1,8)=18.65, p<.005]$.

The mean DR for the third test session (in which novel slides were used) was .51 , which was not significantly different from $.50(t<1)$. Both the center-key and the side-key DRs were significantly higher than the test session DR $[t(4)=3.17$ and 6.38 , respectively; both $p$ s < $.04]$; the different-keys DR was not, however, significantly higher than the test $\mathrm{DR}[t(4)=1.27, p>.2]$.

The results of the two phases of Experiment 4 agreed in that there was considerably better performance in a relative recency task as well as a familiarity/novelty discrimination when the two presentations of an identical image occurred on the same key rather than when those presentations were on different keys. The major contrast between the results of Phases 1 and 2 was that, in Phase 1, the pigeons did show above-chance detection of the identity of familiar images presented on two different keys, but they failed to do so in Phase 2, when novel images were used.

\section{GENERAL DISCUSSION}

The results of the various phases of Experiment 1 are congruent with the conclusion indicated by Phase 2 of Experiment 4-that pigeons in this apparatus do not recognize a novel pictorial image that is projected in two different locations as being the same. These findings do, therefore, provide a resolution of the paradox outlined in the introduction--that pigeons can readily acquire a novelty/familiarity discrimination (using a single key), but show great difficulty in acquiring generalized (N)MTS (in which novel pictorial stimuli are shown on different keys). It is, however, evident from Phase 1 of Experiment 4 that pigeons do recognize familiar pictorial images shown on different keys. The observation in Experiment 3 of an initial preference for a nonmatching color stimulus indicates also that pigeons do detect the identity of novel colors shown on different keys in this apparatus.

One question posed by these findings is the following: Why do pigeons show between-key recognition of novel color stimuli and of familiar pictorial stimuli, but not of novel pictorial stimuli?

An account of the contrast between novel and familiar pictorial stimuli can be provided in terms of a theory of perceptual learning advanced by McLaren, Kaye, and Mackintosh (1989). Their theory supposes that complex visual stimuli consist of elements, and that animals sample only a subset of those elements on a given trial. Associations are formed between elements that are sampled simultaneously and, given the further assumption 
that there will be substantial overlap between the sets of elements activated over a series of trials, a network of excitatory associations will be formed between all the elements of a stimulus that are present on all trials. Elements that are rarely sampled (noise) will form only weak connections with the network, which will, therefore, correspond to the "central tendency" of the stimulus. Thus, the sampling of a subset of the elements will eventually result in the activation of a network that involves representations of all the elements of the stimulus. A major function of this mechanism is to allow animals to identify a repeatedly seen stimulus better than one seen for the first time. This account therefore provides a ready explanation for the finding that pigeons perform more efficiently on a recency discrimination, using familiar stimuli, than on a recognition task, using novel stimuli (e.g., Todd \& Mackintosh, 1990; see also Experiment 4 of the present report). Given the assumption that the subsets of stimuli sampled on a center key vary to some extent from the subsets sampled on a sidekey, this theory can also explain why totally unfamiliar slides are not readily recognized when shown on different keys, and why familiarization of slides results in recognition between keys.

A further relevant aspect of McLaren et al.'s (1989) theory is that it supposes that perceptual learning will not play a significant role in the identification of "simple" stimuli, such as tones and colored lights; this is because "there will be little variability in input from one occasion to the next; in other words, a relatively large proportion of the elements of the stimulus will therefore be sampled on each trial" (McLaren et al., 1989, p. 109). The theory, then, expects that for simple stimuli there will be a substantial overlap between the elements sampled, not only on different trials but also on different keys. It can, then, also explain why plain colored stimuli are readily recognized when shown on different keys.

A second question arises from our data: Why have pigeons shown such poor transfer to novel stimuli following (N)MTS training with either familiar pictorial slides (e.g., Santiago \& Wright, 1984) or plain colored stimuli (e.g., Wilson et al., 1985a)? The most impressive level of transfer reported to date was that obtained by Wright et al. (1988), who used computer-generated images; but in their first transfer test, conducted after more than 18,000 MTS acquisition trials using the same set of 152 trial-unique stimuli each day, 2 pigeons that had achieved a mean accuracy of just below $75 \%$ correct using the familiar stimuli dropped to a level of approximately $62 \%$ correct when entirely novel stimuli were used in a test session. Those same birds did, however, show perfect transfer to a different set of novel stimuli in a subsequent test session carried out after further training (to a total of more than 27,000 trials).

An important feature of the conventional (N)MTS task is that it can readily be solved as a conditional discrimination. A subject may, for example, learn a few simple rules, such as: In the presence of red on the center key, select green on the side key. If identity is, rela- tive to conditional cues, not a salient cue for pigeons, then solving a discrimination as a conditional discrimination may overshadow learning about identity or difference between stimuli. Thus, (N)MTS training using either plain colored stimuli or familiar pictorial stimuli may show little or no transfer to novel stimuli because, although identity is detected by the birds, the task nevertheless is solved as a conditional discrimination (and conventional three-key (N)MTS training using novel stimuli on every trial is very slow to achieve, because pigeons do not readily detect between-key identity of novel pictorial slides).

The proposal that, for pigeons, conditional cues are more salient than identity/difference cues does not imply that pigeons show peculiar difficulty (relative, for example, to individuals of other species) in using identity/ difference as a cue. Wilson, Mackintosh, and Boakes (1985b), for example, found that jackdaws (Corvus monedula), unlike pigeons, learned an MTS task more rapidly following previous MTS training (with different stimuli) than following training in a conditional task. The jackdaws, then, achieved relational learning in the initial MTS task. But the jackdaws showed a poorer absolute level of performance than the pigeons on MTS tasks. Thus, the failure of pigeons to adopt a relational solution can as well (or as badly) be interpreted as showing that jackdaws have a peculiar difficulty in detecting conditional cues as that pigeons have a peculiar difficulty in detecting identity/difference cues. All that can be concluded is that cue saliences vary between the species in such a way that pigeons are more likely than jackdaws to adopt a conditional as opposed to an identity/difference solution when both modes of solution are available.

Difficulty in between-key recognition of stimuli might be expected to have important consequences for many tasks other than (N)MTS. D'Amato, Salmon, Loukas, and Tomie (1985) explored transitivity in monkeys (Cebus apella) and pigeons using a design that consisted of three stages of training. In Stage 1, the animals acquired a conditional discrimination in which two stimuli (A1 and A2) served as center-key sample stimuli, and two other stimuli, B1 and B2, served as side-key comparison stimuli. When A1 was the sample, choice of $B 1$ was rewarded, and when $A 2$ was the sample, choice of B2 was rewarded. In Stage 2, a further conditional discrimination was acquired in which $\mathrm{B} 1$ and $\mathrm{B} 2$ served as sample stimuli, and $\mathrm{C} 1$ and $\mathrm{C} 2$ served as comparison stimuli. In the final stage, A1 and A2 were the sample stimuli and $\mathrm{C} 1$ and $\mathrm{C} 2$ were the comparison stimuli. The monkeys, but not the pigeons, showed a high degree of transfer in the third stage, displaying what D'Amato et al. referred to as "associative transitivity." The monkeys behaved as though A1 (or A2) elicited a representation of $\mathrm{B} 1$ (B2), which in turn elicited a representation of $\mathrm{C} 1$ (C2) and allowed appropriate choice in Stage 3. D'Amato et al. suggested that pigeons, unlike monkeys, are not capable of associative transitivity. As Zentall, Sherburne, Steirn, Randall, and Roper (1992) have 
pointed out, however, successful performance in this test of transitivity requires animals to treat the $\mathrm{B} 1$ and $\mathrm{B} 2$ samples (on the center key) the same as the B1 and B2 comparison stimuli (on the side keys). The present experiments confirm Zentall et al.'s proposal that pigeons do not necessarily generalize between versions of the same stimuli exposed in different locations. Our experiments also provide evidence that perceptual processes are critical in determining whether pigeons readily detect the identity of stimuli shown on different keys. It may, then, be difficult to establish relational learning in certain tasks, not because pigeons are less intelligent than animals that succeed in those tasks, but because pigeons see the world in a somewhat different way.

\section{REFERENCES}

Brown, P. L., \& Jenkins, H. M. (1968). Auto-shaping of the pigeon's key-peck. Journal of the Experimental Analysis of Behavior, 11, 1-8.

Cumming, W. W., \& Berryman, R. (1965). The complex discriminated operant: Studies of matching-to-sample and related problems. In D. I. Mostofsky (Ed.), Stimulus generalization (pp. 284-330). Stanford, CA: Stanford University Press.

D'Amato, M. R., Salmon, D. P., Loukas, E., \& Tomie, A. (1985). Symmetry and transitivity of conditional relations in monkeys (Cebus apella) and pigeons (Columba livia). Journal of the Experimental Analysis of Behavior, 44, 35-47.

FARTHING, G. W., \& OpuDA, M. J. (1974). Transfer of matching-tosample in pigeons. Journal of the Experimental Analysis of Behavior, 21, 199-213.

GinsBURG, N. (1957). Matching in pigeons. Journal of Comparative \& Physiological Psychology, 50, 261-263.

MACKINTOSH, N. J. (1987). Neurobiology, psychology and habituation. Behaviour Research \& Therapy, 25, 81-97.

MaCPHAil, E. M., \& ReILly, S. (1989). Rapid acquisition of a novelty versus familiarity concept by pigeons (Columba livia). Journal of
Experimental Psycholog.: Animal Behavior Processes, 15, 242252.

Milaren, I. P., Kaye, H., \& Mackintosh, N. J. (1989). An associative theory of the representation of stimuli: Applications to perceptual learning and latent inhibition. In R. G. M. Morris (Ed.), Parallel distributed processing. Implications for psuchology and neurobiolog. (pp. 102-130). Oxford: Oxford University Press, Clarendon Press.

MiLlenson, J. R. (1971). A programming language for on-line control of psychological experiments. Behavioral Science, 16, 248-256.

SANTIAGO, H. C., \& WRIGHT, A. A. (1984). Pigeon memory: Same/ different concept learning, serial probe recognition acquisition and probe delay effects in the serial position function. Journal of Experimental Psychology: Animal Behavior Processes, 10, 498-512.

ToDD, I. A., \& MACKINTOSH, N. J. (1990). Evidence for perceptual learning in pigeons' recognition memory for pictures. Quarterly Journal of Experimental Psychology, 42B, 385-400.

Wilson, B., Mackintosh, N. J., \& BoAKes, R. A. (1985a). Matching and oddity learning in the pigeon: Transfer effects and the absence of relational learning. Quarterly Journal of Experimental Psychology, 37B, 295-311.

Wilson, B., Mackintosh, N. J., \& Boakes, R. A. (1985b). Transfer of relational rules in matching and oddity learning by pigeons and corvids. Quarterly Journal of Experimental Psychology, 37B, 313 332.

Wright, A. A., CoOK, R. G., Rivera, J. J., Sands, S. F., \& Delius, J. D. (1988). Concept learning by pigeons: Matching-to-sample with trial-unique video picture stimuli. Animal Learning \& Behavior, 16, 436-444.

ZENTALL, T. R., \& HOGAN, D. E. (1974). Abstract concept learning in the pigeon. Journal of Experimental Psychology, 102, 393-398.

Zentall, T. R., Sherburne, L. M., Steirn, J. N., Randall, C. K., \& ROPER, K. L. (1992). Common coding in pigeons: Partial versus total reversals of one-to-many conditional discriminations. Animal Learning \& Behavior, 20, 373-381.

(Manuscript received July 8, 1993; revision accepted for publication March 31, 1994.) 